\title{
Nonlinear Dynamical Analysis for the Cable Excited with Parametric and Forced Excitation
}

\author{
C. Z. Qian, C. P. Chen, and G. W. Zhou \\ College of Civil Engineering and Architecture, Xiamen University of Technology, Xiamen, Fujian 361024, China \\ Correspondence should be addressed to C. Z. Qian; qiancz@163.com
}

Received 10 April 2014; Revised 20 August 2014; Accepted 7 September 2014; Published 19 October 2014

Academic Editor: Ali R. Ashrafi

Copyright (C) 2014 C. Z. Qian et al. This is an open access article distributed under the Creative Commons Attribution License, which permits unrestricted use, distribution, and reproduction in any medium, provided the original work is properly cited.

Considering the deck vibration effect on the cable in cable-stayed bridge, using nonlinear structure dynamics theory, the nonlinear dynamical equation for the stayed cable excited with deck vibration is proposed. Research shows that the vertical vibration of the deck has a combined parametric and forced excitation effect on the cable when the angle of the cable is taken into consideration. Using multiscale method, the $1 / 2$ principle parametric resonance is studied and the bifurcation equation is obtained. Despite the parameters analysis, the bifurcation characters of the dynamical system are studied. At last, by means of numerical method and software MATHMATIC, the effect rules of system parameters to the dynamical behavior of the system are studied, and some useful conclusions are obtained.

\section{Introduction}

Along with the new materials, new technology continues to improve, large span cable is applied widespread, and the cable-stayed bridge becomes one of the main forms of longspan bridges in the word. Because of the flexibility, quality, and small damping characteristic, the dynamical problem of the cable structure is relatively prominent. In recent decades, the dynamics of stayed cable has gained widespread concern in the bridge engineering. Researches have shown that the breeze and light rain conditions may induce the cable having dramatic large amplitude vibrations [1-4]. Such sharp vibration may cause the fatigue fracture of cable or junction of fatigue cracks in the cable anchor, undermine the claim of anticorrosion system, and cause harm to the safety and durability of the bridge.

In the earlier 1970s, some researchers began to study the cable vibration in cable-stayed bridge [5]. Variety induced vibration theory is proposed, such as vibration induced with rain and wind, secondary axial flow vibration, threedimensional vortex shedding, and wake galloping. But so far stayed cable vibration mechanism was not studied clearly. It is shown that the cable vibration is a complex phenomenon affected by multiple factors. The aerodynamic characteristics caused by the wind and rain and the geometric nonlinearity of the bridge structure. The parametric vibration about cable is studied in the literature [6], in which a single mass is used to simulate continue cable. The parametric vibration about cable is also studied in literature [7], in which the cable is considered a vertical chord. Considering the initial deflection, the tension, and external excitation, the coupling dynamics of the cable about parametric vibration and forced vibration is studied [8].

The vertical direction vibration of the deck will be induced with the wind loads or other live loads role. When an order of the natural frequency of the deck is similar to the twice the cable's natural frequency, slight vibration of the bridge deck will inspire cable vibration sharply [9, 10]. This phenomenon is significant feature of the parametric vibration. Therefore, studying this vibration problem is meaningful to design and protection of the cable structure. In the literature [11], the equations governing the linearized dynamics of the cable-stayed beam are presented and the main features of the parametric vibration phenomenon are investigated. The literature [12] investigates the coupled nonlinear vibration behaviors of the cable/deck system by numerical analysis of a proposed three-degree-of-freedom model and using the Runge-Kutta integration method reveal some fundamental characteristics of the nonlinear cable vibration. 
Parametric vibration system is the incentive depending on the time and appears as a parameter in the control equation. A feature of such a vibration system is the system parameter with the time change. When the natural frequency of the system has a multiple relationship with the excitation frequency, even a small excitation can cause complex responses $[13,14]$ of the system which is different from the external excitation.

Based on the literature [6], using bifurcation theory and nonlinear vibration theory, the coupling effect on parametric excitation and external force to the cable vibration is studied. Using numerical method, the bifurcation response is obtained, in which some nonlinear phenomenon is shown with figures.

\section{Nonlinear Dynamical Equation of the Stay Cable}

In order to study vibration mechanism of the stay cable bridge, the cable vibration system is modeled as Figure 1. In this mechanics model, the initial deflection and off-plane motion are not considered. Using nonlinear vibration theory [13], the dynamical equation of the cable in plan $x y$ is expressed as (1). Consider

$$
\begin{aligned}
& \rho A \frac{\partial^{2} w}{\partial t^{2}}-T \frac{\partial^{2} w}{\partial x^{2}}+2 \mu \frac{\partial w}{\partial t} \\
& \quad=\frac{E A}{L}\left[D(t) \cos \gamma+\frac{1}{2} \int_{0}^{L}\left(\frac{\partial w}{\partial x}\right)^{2} d x\right] \frac{\partial^{2} w}{\partial x^{2}} .
\end{aligned}
$$

With the boundary condition

$$
w(0, t)=0, \quad w(L, t)=D(t) \sin \gamma,
$$

in which $w$ denotes the displacement of cable in $y$ direction, $L$ denotes the length, $A$ is the section area of the cable, $E$ denotes elastic modulus, $\rho$ denotes the density of the material, and $\gamma$ is the angle between cable and vertical direction. $D(t)=$ $D \cos \omega t$ is the vertical displacement of the connection point of the cable and the bridge deck. If only the first order modal is considered, the solution of (1) can be denoted by

$$
w(x, t)=\frac{x}{L} D(t) \sin \gamma+y(t) \sin \frac{\pi x}{L} .
$$

For purpose of analysis, some nondimensional parameters are introduced as follows:

$$
\begin{aligned}
& \widehat{w}=\frac{w}{L}, \quad d=\frac{D}{L}, \quad \eta=\frac{x}{L}, \quad \tau=\omega t, \\
& \omega_{0}=\frac{\pi}{L} \sqrt{\frac{T}{\rho A}}, \quad \lambda=\frac{\omega_{0}}{\omega}, \quad c=\frac{\pi}{\omega L} \sqrt{\frac{E}{\rho} .}
\end{aligned}
$$

Then (1)-(3) can be rewritten as

$$
\begin{gathered}
\frac{\partial^{2} \widehat{w}}{\partial \tau^{2}}-\frac{T}{\rho A L^{2} \omega^{2}} \frac{\partial^{2} \widehat{w}}{\partial \eta^{2}}+\frac{2 \mu}{\rho A L \omega} \frac{\partial \widehat{w}}{\partial \tau} \\
=\frac{E}{\rho L^{2} \omega^{2}}\left[d \cos \tau \cos \gamma+\frac{1}{2} \int_{0}^{1}\left(\frac{\partial \widehat{w}}{\partial \eta}\right)^{2} d \eta\right] \frac{\partial^{2} \widehat{w}}{\partial \eta^{2}}, \\
\widehat{w}(0, \tau)=0, \quad \widehat{w}(1, \tau)=d \cos \tau \sin \gamma, \\
\widehat{w}(\eta, \tau)=\eta d \cos \tau \sin \gamma+y(\tau) \sin \pi \eta .
\end{gathered}
$$

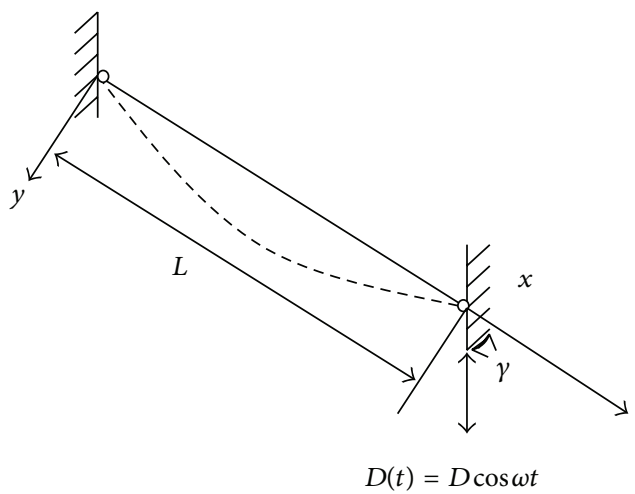

FIgURe 1: Dynamical modules of cable.

Substituting (7) into (5) and adding the viscous damping item, using Galerkin integration method, the differential equation about $y(t)$ can be simplified as

$$
\begin{aligned}
& \frac{d^{2} y}{d \tau^{2}}+2 \xi \frac{d y}{d \tau}+\left[c_{1}^{2}+c_{2}^{2}\left(d \cos \tau \cos \gamma+\frac{1}{2} d^{2} \cos ^{2} \tau \sin ^{2} \gamma\right)\right] \\
& \quad \times y(\tau)+\frac{c_{2}^{2}}{2} y^{3}(\tau)-\frac{2 d}{\pi} \sin \gamma \cos \tau=0
\end{aligned}
$$

when $\gamma=0$ and (8) has not forced vibration item, which means the system (1) has only parametric vibration and this case has been introduced in literature [6]. In general case, $\gamma \neq 0$, forced vibration and parametric vibration items exist in (8) at the same time and system (1) should be considered a dynamical system with combined forced and parametric excitation. Considering $d^{2}$ is higher order small quantities than $d,(1 / 2) d^{2} \cos ^{2} \tau \sin ^{2} \gamma$ can be ignored and (8) can be simplified as

$$
\begin{gathered}
\frac{d^{2} y}{d \tau^{2}}+2 \xi \frac{d y}{d \tau}+\left[\lambda^{2}+c_{2}^{2} d \cos \tau \cos \gamma\right] y(\tau) \\
+\frac{c_{2}^{2}}{2} y^{3}(\tau)-\frac{2}{\pi} d \sin \gamma \cos \tau=0 .
\end{gathered}
$$

\section{Bifurcation Response Equations}

In order to take advantage of multiscale method analysis, ignoring the effect of deflect and using the substitution parameters,

$$
\begin{aligned}
\xi \longrightarrow \varepsilon \mu, & \frac{c_{2}^{2}}{2} \longrightarrow \varepsilon^{2} \beta, \quad c_{2}^{2} d \cos \gamma \longrightarrow \varepsilon f, \\
& -\frac{2 d}{\pi} \sin \gamma \longrightarrow p .
\end{aligned}
$$

Then, (9) can be rewritten as

$$
\frac{d^{2} y}{d \tau^{2}}+2 \varepsilon \mu \frac{d y}{d \tau}+\lambda^{2} y+\varepsilon^{2} \beta y^{3}+\varepsilon f y \cos \tau+p \cos \tau=0 .
$$


Based on the multiple scale method, the perturbation solution of (11) can be expensed as the power series about small parameter $\varepsilon$ :

$$
\begin{aligned}
y(\varepsilon, \tau)= & y_{0}\left(T_{0}, T_{1}, T_{2}\right)+\varepsilon y_{1}\left(T_{0}, T_{1}, T_{2}\right) \\
& +\varepsilon^{2} y_{2}\left(T_{0}, T_{1}, T_{2}\right)+\cdots,
\end{aligned}
$$

in which $T_{i}=\varepsilon^{i} \tau$. Considering the differential connections,

$$
\frac{d}{d \tau}=\frac{\partial}{\partial T_{0}}+\varepsilon \frac{\partial}{\partial T_{1}}+\varepsilon^{2} \frac{\partial}{\partial T_{2}}=D_{0}+\varepsilon D_{1}+\varepsilon^{2} D_{2} .
$$

Substituting (12) into (11), a series perturbation equations can be obtained as follows:

$$
\begin{gathered}
D_{0}^{2} y_{0}+\lambda^{2} y_{0}=-p \cos T_{0}, \\
D_{0}^{2} y_{1}+\lambda^{2} y_{1}=-2 D_{0} D_{1} y_{0}-2 \mu D_{0} y_{0}-f y_{0} \cos T_{0}, \\
D_{0}^{2} y_{2}+\lambda^{2} y_{2}=-2 D_{0} D_{2} y_{0}-D_{1}^{2} y_{0}-2 D_{0} D_{1} y_{1} \\
-2 \mu D_{0} y_{1}-2 \mu D_{1} y_{0}-\beta y_{0}^{3}-f y_{1} \cos T_{0} .
\end{gathered}
$$

Solving (14), $y_{0}$ can be expressed as

$$
y_{0}=A\left(T_{1}, T_{2}\right) e^{i \lambda T_{0}}+\bar{A}\left(T_{1}, T_{2}\right) e^{-i \lambda T_{0}}+G\left(e^{i T_{0}}+e^{-i T_{0}}\right),
$$

in which $G=p / 2\left(1-\lambda^{2}\right)$. Substituting (17) into (15), then (15) can be rewritten as

$$
\begin{aligned}
D_{0}^{2} y_{1}+\lambda^{2} y_{1}= & -2 i \lambda D_{1} A e^{i \lambda T_{0}}+2 i \lambda D_{1} \bar{A} e^{-i \lambda T_{0}} \\
& -2 i \mu \lambda A e^{i \lambda T_{0}}+2 i \mu \lambda \bar{A} e^{-i \lambda T_{0}} \\
& -2 i \mu G e^{i T_{0}}+2 i \mu G e^{-i T_{0}} \\
& -\frac{f}{2} A e^{i(\lambda+1) T_{0}}-\frac{f}{2} \bar{A} e^{-i(\lambda+1) T_{0}} \\
& -\frac{f}{2} G e^{2 i T_{0}}-f G-\frac{f}{2} \bar{A} e^{-i(\lambda-1) T_{0}} \\
& -\frac{f}{2} A e^{i(\lambda-1) T_{0}}-\frac{f}{2} G e^{-2 i T_{0}} .
\end{aligned}
$$

Considering the $1 / 2$ main parametric resonance condition, assuming

$$
\lambda=\frac{\omega_{0}}{\omega}=\frac{1}{2}-\varepsilon \sigma
$$

in which $\sigma$ is tuning parameter, substituting (19) into (18), and eliminating the long-term items, then (20) is obtained as follows:

$$
2 i \lambda D_{1} A+2 i \mu \lambda A+\frac{f}{2} \bar{A} e^{2 i \sigma T_{1}}=0 .
$$

Solving (18), $y_{1}$ can be obtained as

$$
\begin{aligned}
y_{1}= & \frac{2 i \mu G}{1-\lambda^{2}} e^{i T_{0}}-\frac{2 i \mu G}{1-\lambda^{2}} e^{-i T_{0}} \\
& +\frac{f A}{2(2 \lambda+1)} e^{i(\lambda+1) T_{0}}+\frac{f G}{2\left(4-\lambda^{2}\right)} e^{2 i T_{0}} \\
& -\frac{f G}{\lambda^{2}}+\frac{f \bar{A}}{2(2 \lambda+1)} e^{-i(\lambda+1) T_{0}}+\frac{f G}{2\left(4-\lambda^{2}\right)} e^{-2 i T_{0}} .
\end{aligned}
$$

Substituting (17), (21) into (16) and eliminating the long-term items, then (22) is obtained as follows:

$$
\begin{aligned}
- & 2 i \lambda D_{2} A-D_{1}^{2} A-2 \mu D_{1} A-\beta\left(3 A^{2} \bar{A}+6 G^{2} A\right) \\
- & \frac{f^{2} A}{4(2 \lambda+1)}=0 .
\end{aligned}
$$

Associating (20), eliminating the $D_{1}^{2} A$ and $D_{1} A$ items from (22), and considering $2 \lambda \approx 1$, then (23) can be obtained as follows:

$$
i D_{2} A-\mu^{2} A-f \sigma \bar{A} e^{2 i \sigma T_{1}}+\beta\left(3 A^{2} \bar{A}+6 G^{2} A\right)+\frac{3 f^{2} A}{8}=0 .
$$

Considering the differential connection,

$$
\frac{d A}{d \tau}=\varepsilon D_{1} A+\varepsilon^{2} D_{2} A
$$

Equation (24) can be rewritten as

$$
\begin{aligned}
& i\left(\frac{d A}{d \tau}+\varepsilon \mu A\right)+\frac{1}{2} \varepsilon f \bar{A} e^{2 i \sigma T}-\varepsilon^{2} \\
& \times\left[\mu^{2} A-f \sigma \bar{A} e^{2 i \sigma T_{1}}+\beta\left(3 A^{2} \bar{A}+6 G^{2} A\right)+\frac{3 f^{2} A}{8}\right]=0 .
\end{aligned}
$$

Assuming that $A=(1 / 2) a(\tau) e^{i \varphi(\tau)}$ and is substituted into (25), separating the real part and the imaginary part, (26) can be obtained as

$$
\begin{aligned}
\frac{d a}{d \tau}= & -\varepsilon \mu a-\left(\frac{1}{2} \varepsilon f+f \sigma \varepsilon^{2}\right) a \sin \theta \\
\frac{1}{2} a \frac{d \theta}{d \tau}= & 2 \varepsilon \sigma+2 \varepsilon^{2} \mu^{2} a-12 \varepsilon^{2} \beta G^{2} a \\
& -6 \varepsilon^{2} \beta a^{3}-\left(\varepsilon f-2 f \sigma \varepsilon^{2}\right) a \cos \theta
\end{aligned}
$$

where

$$
\theta=\varepsilon \sigma \tau-2 \varphi
$$

For $d a / d \tau=d \theta / d \tau=0$ corresponding to the steady-state solution of the system, (26) can be rewritten as

$$
\begin{gathered}
\varepsilon \mu a+\left(\frac{1}{2} \varepsilon f+f \sigma \varepsilon^{2}\right) a \sin \theta=0, \\
-\varepsilon \sigma \frac{a}{2}-\varepsilon^{2} \mu^{2} a+6 \varepsilon^{2} \beta G^{2} a+3 \varepsilon^{2} \beta a^{3} \\
+\left(\frac{1}{2} \varepsilon f-f \sigma \varepsilon^{2}\right) a \cos \theta=0 .
\end{gathered}
$$


Eliminating $\theta$ from (28), then

$$
\begin{aligned}
\mu^{2} a^{2} & +\left(\frac{1}{2} \sigma-\varepsilon \mu^{2}+6 \varepsilon \beta G^{2}+3 \varepsilon \beta a^{2}\right)^{2} a^{2} \\
& =\left(\frac{1}{2} f-f \sigma \varepsilon\right)^{2} a^{2} .
\end{aligned}
$$

In addition to ordinary solution $a=0$, the nonordinary solution of (29) meets the following equation:

$$
\mu^{2}+\left(\frac{1}{2} \sigma-\varepsilon \mu^{2}+6 \varepsilon \beta G^{2}+3 \varepsilon \beta a^{2}\right)^{2}=\left(\frac{1}{2} f-f \sigma \varepsilon\right)^{2} .
$$

Solving (30), the nonordinary solution can be obtained as

$$
=\frac{-\left((1 / 2) \sigma-\varepsilon \mu^{2}+6 \varepsilon \beta G^{2}\right) \pm\left[((1 / 2) f-f \sigma \varepsilon)^{2}-\mu^{2}\right]^{1 / 2}}{3 \varepsilon \beta} .
$$

Considering different parameters, there may exist some possible solutions as follows:

(I) $((1 / 2) f-f \sigma \varepsilon)^{2}-\mu^{2}=0,(1 / 2) \sigma-\varepsilon \mu^{2}+6 \varepsilon \beta G^{2}<0$, and system has one nonzero solution;

(II) $((1 / 2) f-f \sigma \varepsilon)^{2}-\mu^{2}>0,(1 / 2) \sigma-\varepsilon \mu^{2}+6 \varepsilon \beta G^{2}<$ $0,\left((1 / 2) \sigma-\varepsilon \mu^{2}+6 \varepsilon \beta G^{2}\right)^{2}>((1 / 2) f-f \sigma \varepsilon)^{2}-\mu^{2}$, and system has only two nonzero real solutions;

(III) $((1 / 2) f-f \sigma \varepsilon)^{2}-\mu^{2}>0,(1 / 2) \sigma-\varepsilon \mu^{2}+6 \varepsilon \beta G^{2}<$ $0,\left((1 / 2) \sigma-\varepsilon \mu^{2}+6 \varepsilon \beta G^{2}\right)^{2} \leq((1 / 2) f-f \sigma \varepsilon)^{2}-\mu^{2}$, and system has only one nonzero real solution;

(IV) $((1 / 2) f-f \sigma \varepsilon)^{2}-\mu^{2}>0,(1 / 2) \sigma-\varepsilon \mu^{2}+6 \varepsilon \beta G^{2}>$ $0,\left((1 / 2) \sigma-\varepsilon \mu^{2}+6 \varepsilon \beta G^{2}\right)^{2}>((1 / 2) f-f \sigma \varepsilon)^{2}-\mu^{2}$, and system has only one nonzero real solution;

(V) other conditions, system exist only one zero solution.

From the above-mentioned discussion, it is sure that there are critical excitation amplitude $f_{\text {crit }}$ and fold bifurcation point $f_{T}$. In order to avoid saddle-node bifurcation, the critical bifurcation point $f_{\text {crit }}$ is important. Consider

$$
f_{\text {crit }}= \pm \frac{\mu}{\varepsilon \omega} \text {. }
$$

At this time, the system parameters meet with

$$
\left(\frac{1}{2} \sigma-\varepsilon \mu^{2}+6 \varepsilon \beta G^{2}\right)^{2}=\left(\frac{1}{2} f_{T}-f_{T} \sigma \varepsilon\right)^{2}-\mu^{2}
$$

The supercritical bifurcation is decided with bifurcation point number $f_{T}$ in (33).

\section{Numerical Analysis for the Cable Vibration}

In this section, the numerical method and software MATHMATIC are used to solve a cable system. It aims at analyzing the vibration frequency of deck and inclination angle of the cable effect on the cable vibration. The parameters of the structure are defined as Table 1 , in which the damping is

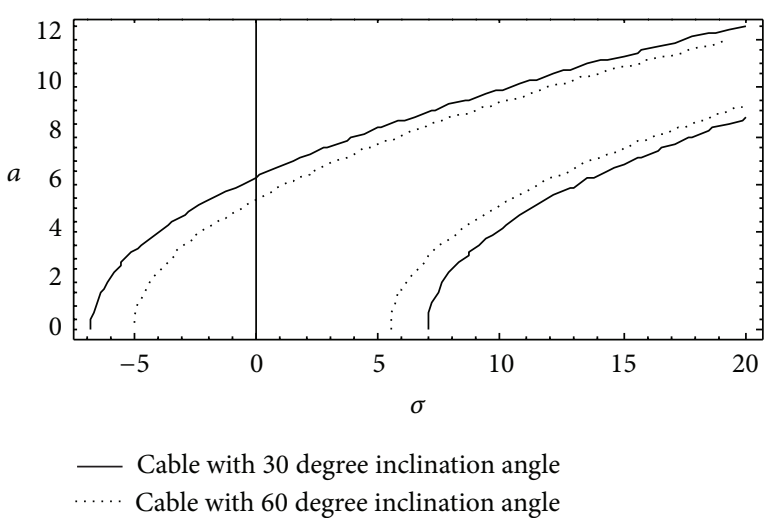

FIGURE 2: Frequency-amplitude curves with various inclination angles of the cables.

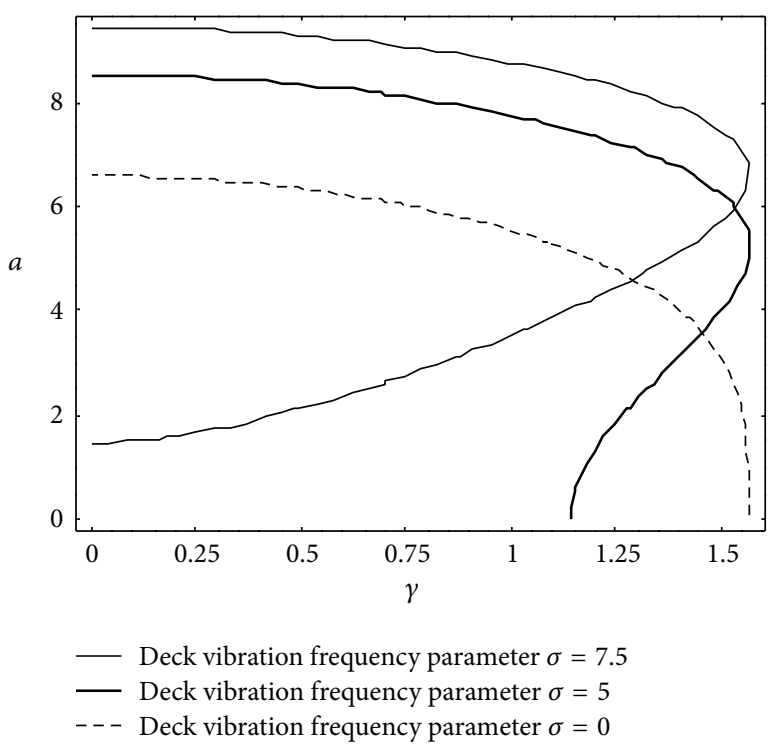

FIGURE 3: Amplitude-inclination angle curves with various deck vibration frequencies.

TABLE 1: The essential parameters of the cable.

\begin{tabular}{lc}
\hline Mass per unit length $(\rho A)$ & $0.391 \mathrm{~kg} / \mathrm{m}$ \\
Length of cable $(L)$ & $11.90 \mathrm{~m}$ \\
Axial tensile force $(T)$ & $1.5 \mathrm{kN}$ \\
Section area $(A)$ & $7.28 \mathrm{~mm}^{2}$ \\
Elastic modulus $(E)$ & $8.242 \times 104 \mathrm{MPa}$ \\
Extensional rigidity $(E A)$ & $6.0 \times 105 \mathrm{~N}$ \\
\hline
\end{tabular}

not considered and the vibration amplitude of the anchorage point is defined as $D=5 \mathrm{~mm}$. the first natural vibration frequency is obtained $f=2.6 \mathrm{~Hz}$ and $\omega_{0}=16.352$.

Using the numerical method, the bifurcation equation (29) and the different equation (9) are solved, in which the deck vibration amplitude is assumed as constant. The parametric resonance bifurcation curves corresponding to various inclination angles of the cables can be obtained as Figure 2. 


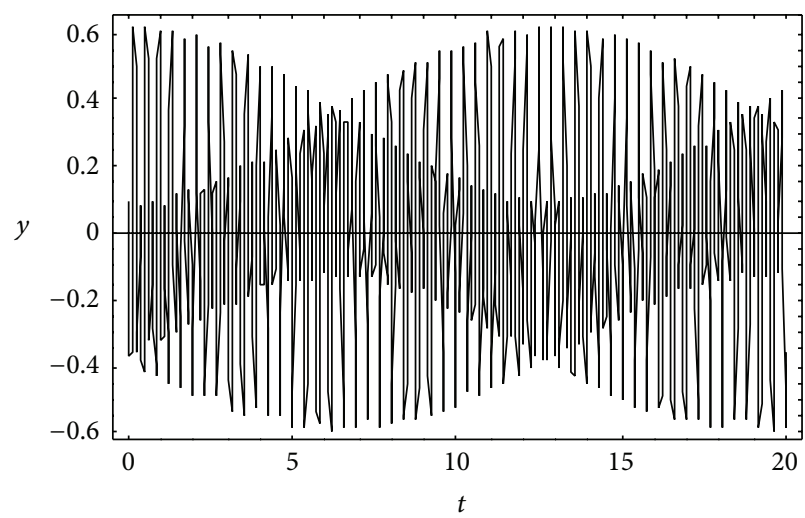

(a)

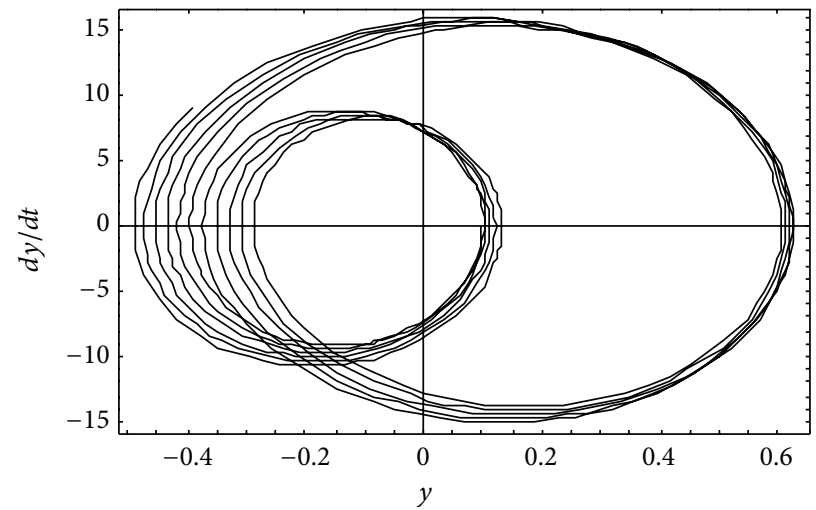

(b)

Figure 4: Time domain curve and phase plane about the systems $\sigma=0, \gamma=\pi / 3$.

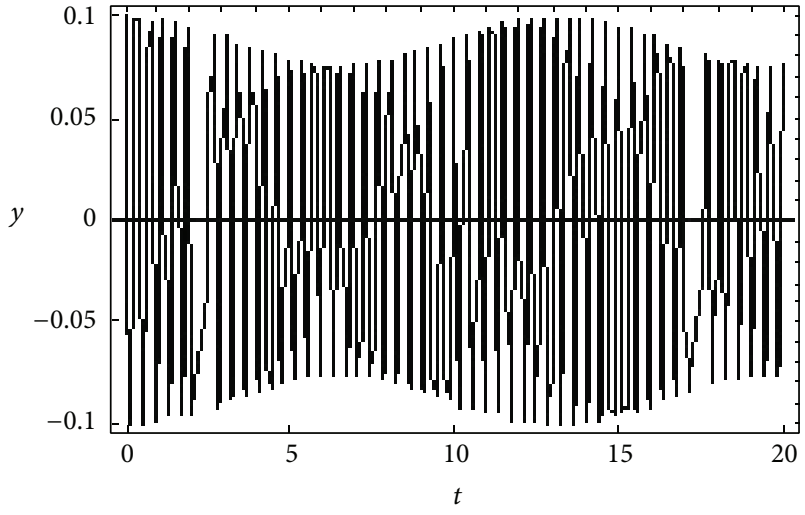

(a)

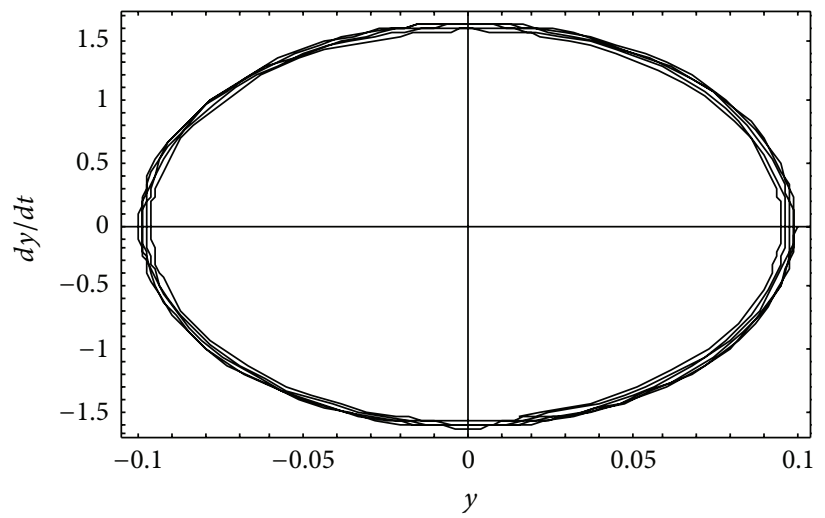

(b)

Figure 5: Time domain curve and phase plane about the systems $\sigma=0, \gamma=\pi / 6$.

Results of bifurcation points of parametric resonance corresponding to various cables' inclination angles are given in Figure 2. It shows that inclination angles change the dislocation of bifurcation points.

When the inclination angles of the cable are made small, two bifurcation points become closer and the amplitude of resonance becomes larger. The parametric resonance becomes a dominant factor compared to the forced oscillation. In order to observe the effect of the inclination angle of amplitude response, the amplitude-inclination angle curves are given in Figure 3; in those curves, the vibration frequencies of the anchor point are fixed.

From Figure 3, it can be found that tune frequency parameter $\sigma$ affects not only the bifurcation point values but also the numbers of bifurcation points. Based on the numerical analyzing, the rules are found as follows. (1) When $\sigma \leq 0$, the system has only one critical bifurcation point and the system has only one steady state response solution. (2) The nonsteady response solution occurred as $\sigma$ increase, and a fold bifurcation occurred. (3) When $\sigma$ reach a fixed value, the systems' response changed to be more complicated.
In order to observe the effect of parametric excitation, the curves time domain and phase plan result of some systems are given in Figures 4 and 5.

From Figures 4 and 5, some interesting phenomenon is observed. As the case of $0<\gamma<\pi / 2$, the vibration responses are caused by parametric excitation and forced excitation or the corresponding joint action. As $\gamma$ decreases, the parametric excitation plays a major role; this conclusion meets well with [8]. When the value of $\gamma$ is larger, parametric excitation and forced excitation combined effects are obvious; its dynamic behavior is more complex and will need further research. So, this paper will not repeat them.

\section{Conclusion}

In this paper, nonlinear dynamical characters of the cable in cable-stayed bridge are studied. First, using nonlinear structure dynamics, a nonlinear model of a cable in cablestayed bridge excited with deck vibration is proposed. Secondly, using multiscale method, the $1 / 2$ principle parametric 
resonance is studied and the bifurcation equation is obtained. And then the bifurcation characters of the dynamical system are studied. At last, the effect rules of system parameters to the dynamic behavior of the system are studied. Through study of this dynamical system, some useful conclusions are obtained.

(1) Parametric vibration of the cables in the cable-stayed bridge is induced with the vertical vibration of the deck. At the same time, the vertical vibration of the deck has the forced excitation effect on the cables.

(2) In general, the vibration response of the cable is joint effect of the parametric and forced excitation.

(3) As $\gamma$ decreases, the parametric excitation plays a major role. When the value of $\gamma$ is larger, parametric excitation and forced excitation combined effects are obvious.

\section{Conflict of Interests}

The authors declare that there is no conflict of interests regarding the publication of this paper.

\section{Acknowledgment}

This work is supported by the National Science Foundation of China (Grants nos. 51108047, 11272270, and 11102028), Science and Technology Project in Xiamen (Grant no. 3502Z20143028), and XMUT Sino-Foreign Cooperation Foundation (Grant no. E201301500).

\section{References}

[1] M. Matsumoto, N. Shiraishi, and H. Shirato, "Rain-wind induced vibration of cables of cable-stayed bridges," Journal of Wind Engineering \& Industrial Aerodynamics, vol. 43, no. 1-3, pp. 2011-2022, 1992.

[2] Y. Hikami and N. Shiraishi, "Rain-wind induced vibrations of cables stayed bridges," Journal of Wind Engineering and Industrial Aerodynamics, vol. 29, no. 1-3, pp. 409-418, 1988.

[3] J. L. Lilien and A. P. Da Costa, "Vibration amplitudes caused by parametric excitation of cable stayed structures," Journal of Sound and Vibration, vol. 174, no. 1, pp. 69-90, 1994.

[4] H. Yamaguchi and Y. Fujino, "Stayed cable dynamics and its vibration control," in Proceedings of the International Symposium on Advances in Bridge Aerodynamics, pp. 235-253, Balkema Rotterdam, Rotterdam, The Netherlands, 1998.

[5] G. Tagata, "Harmonically forced, finite amplitude vibration of a string," Journal of Sound and Vibration, vol. 51, no. 4, pp. 483492, 1977.

[6] Y. Fujino, P. Warnitchai, and B. M. Pacheco, "Experimental and analytical study on internal resonances in a cable-stayed beam model," Proceedings of Japan Society of Civil Engineering, vol. 432, pp. 109-118, 1991.

[7] Y. Fujino, P. Warnitchai, and B. M. Pacheco, "An experimental and analytical study of autoparametric resonance in a $3 \mathrm{DOF}$ model of cable-stayed-beam," Nonlinear Dynamics, vol. 4, no. 2, pp. 111-138, 1993.

[8] Z. Wang and B. Sun, "Parametric vibration of the cable in cable-stayed bridge," China Civil Engineering Journal, vol. 35, no. 5, pp. 14-22, 2002.
[9] A. Pinto Da Costa, J. A. C. Martins, F. Branco, and J. L. Lilien, "Oscillations of bridge stay cables induced by periodic motions of deck and/or towers," Journal of Engineering Mechanics, vol. 122, no. 7, pp. 613-621, 1996.

[10] N. C. Perkins, "Modal interactions in the non-linear response of elastic cables under parametric/external excitation," International Journal of Non-Linear Mechanics, vol. 27, no. 2, pp. 233250, 1992.

[11] V. Gattulli and M. Lepidi, "Localization and veering in the dynamics of cable-stayed bridges," Computers and Structures, vol. 85, no. 21-22, pp. 1661-1678, 2007.

[12] Z. Kang, K. Xu, and Z. Luo, "A numerical study on nonlinear vibration of an inclined cable coupled with the deck in cablestayed bridges," Journal of Vibration and Control, vol. 18, no. 3, pp. 404-416, 2012.

[13] A. H. Nayfeh and D. T. Mook, Nonlinear Oscillations, John Wiley \& Sons, New York, NY, USA, 1973.

[14] A. H. Nayfeh, Perturbation Method, John Wiley \& Sons, New York, NY, USA, 1973. 


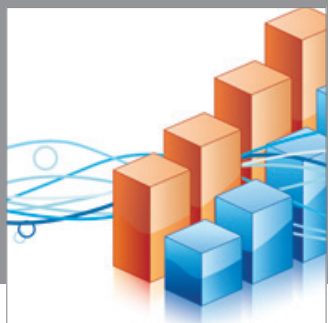

Advances in

Operations Research

mansans

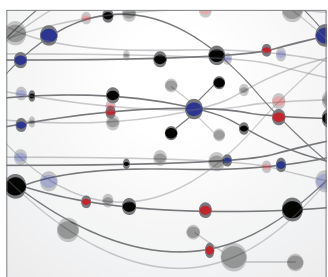

The Scientific World Journal
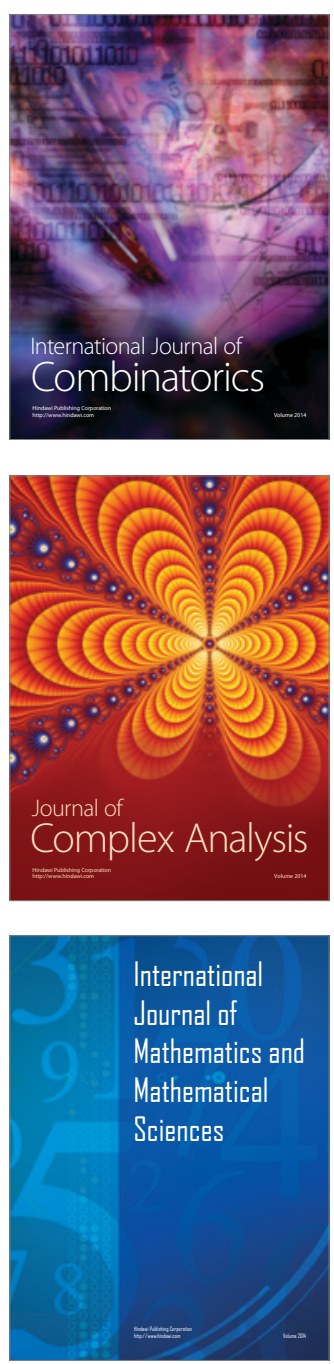
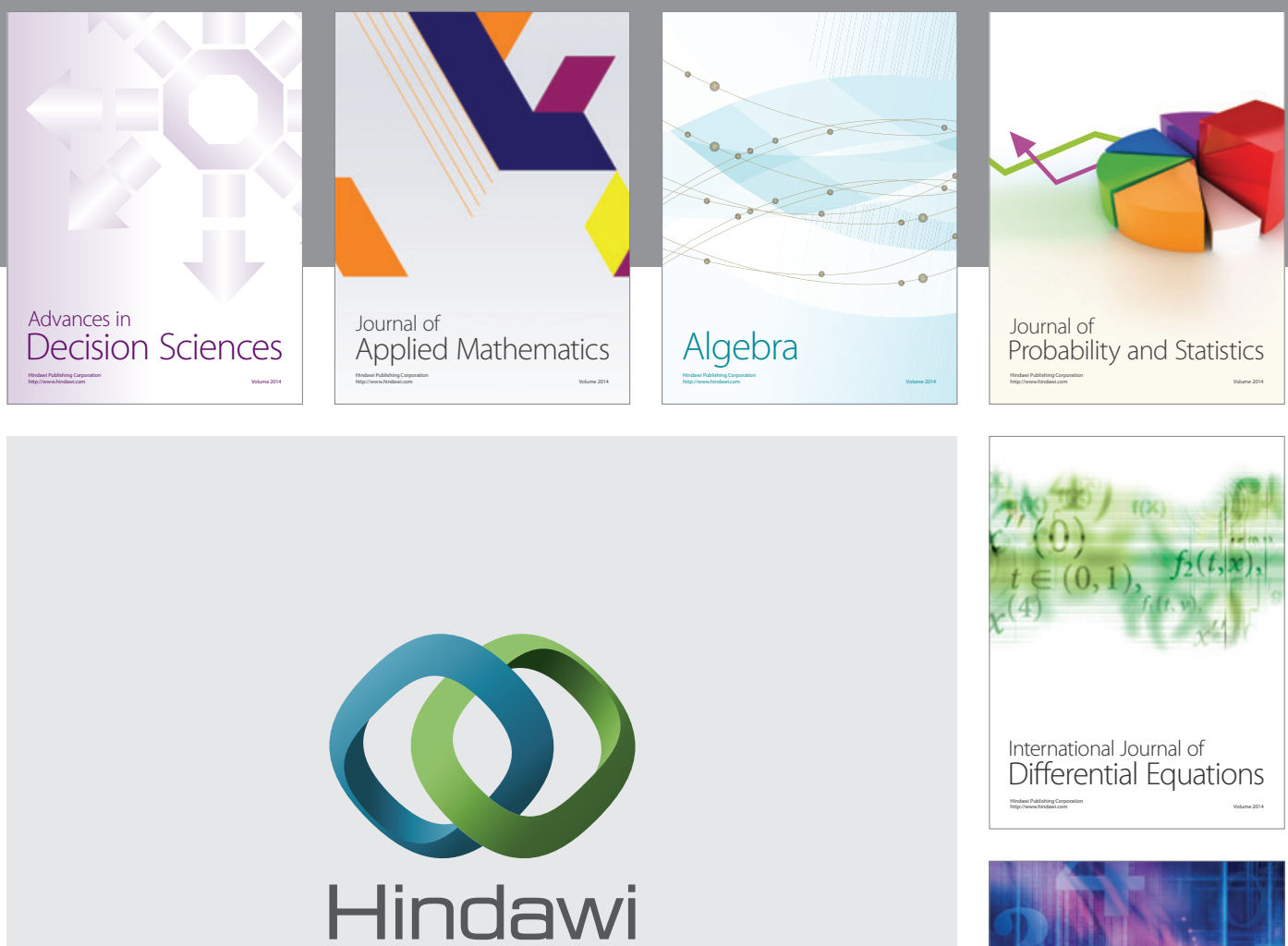

Submit your manuscripts at http://www.hindawi.com
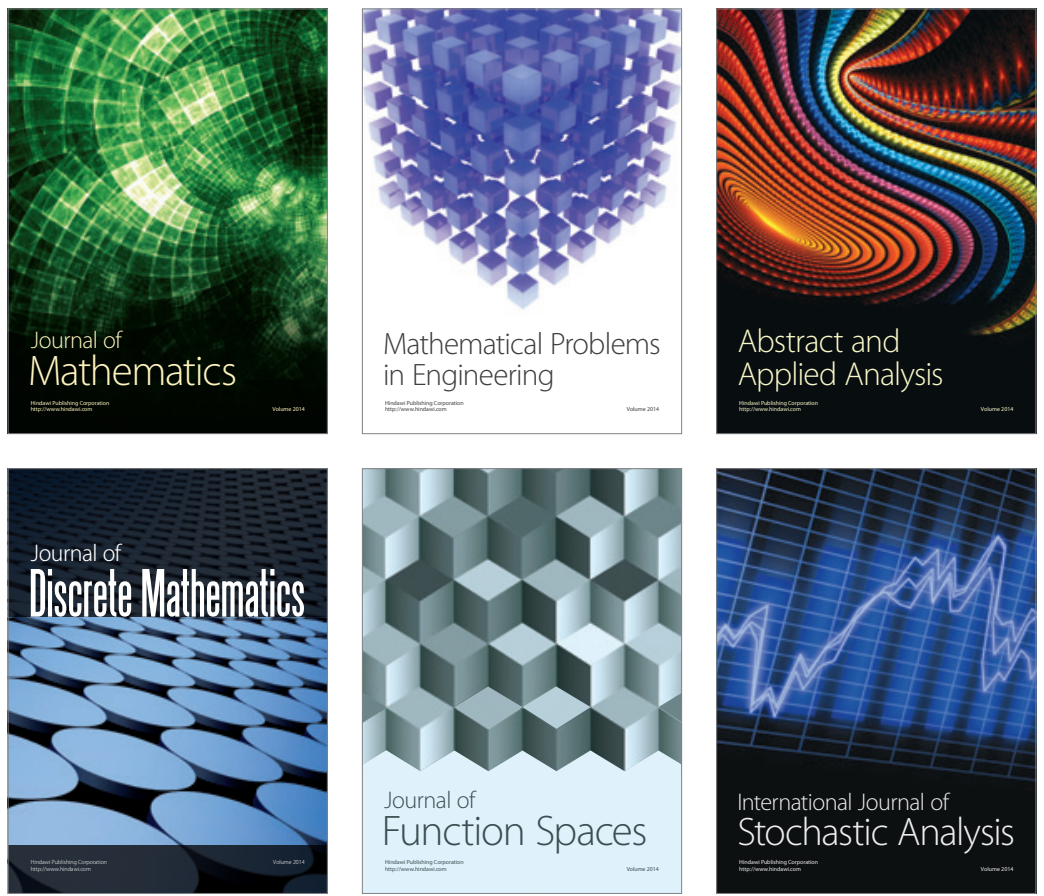

Journal of

Function Spaces

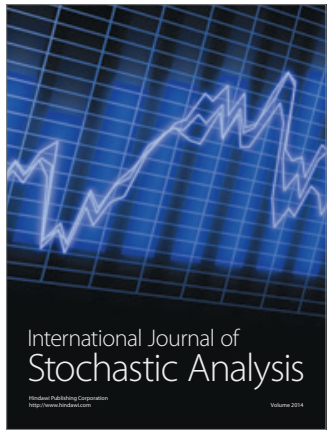

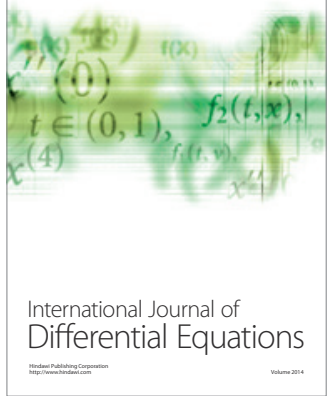
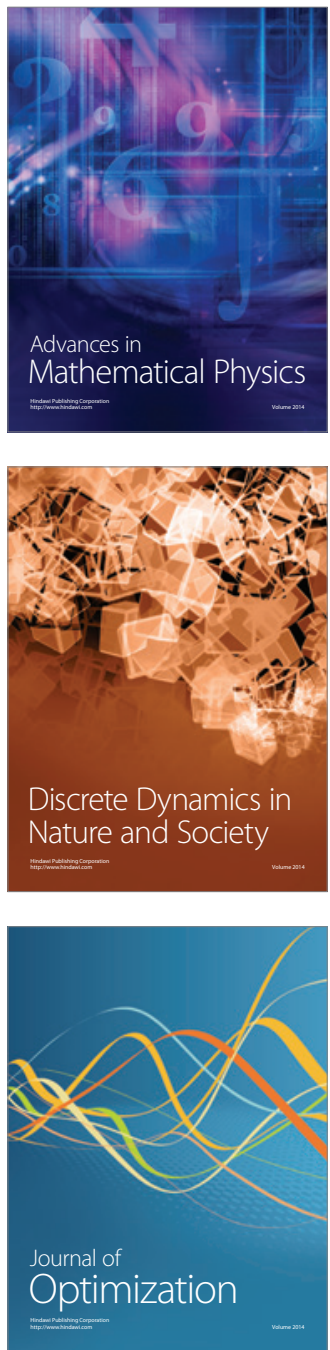